\title{
CAMINANDO ENTRE MEDIDAS INVARIANTES Y TOPOLOGÍAS: EL TEOREMA DE HAAR INVERSO
}

\author{
SAÚL PILATOWSKY CAMEO
}

\begin{abstract}
RESUMEN. En este artículo presentamos el teorema de Haar inverso, originalmente demostrado por Weil en 1940. El teorema de Haar garantiza que en grupos equipados con cierta topología, siempre existe una medida que no cambia al trasladarla por medio de la operación del grupo, es decir, una medida invariante. Esta medida - llamada medida de Haar- guarda una relación estrecha con la topología del grupo. El teorema de Haar inverso garantiza que, en cierto sentido, todas las medidas invariantes son medidas de Haar. Dado un grupo -sin topologíay una medida invariante, se puede construir una topología de manera que la medida invariante es, esencialmente, la medida de Haar.
\end{abstract}

\section{INTRODUCCIÓN}

Detrás del teorema de Haar está la idea de compatibilidad de estructuras. Cuando se estudian espacios que poseen diferentes estructuras a la vez -algebraicas, topológicas y de medida-, se busca imponer condiciones sobre ellos que las hagan compatibles unas con las otras. Por ejemplo, si se tiene un grupo equipado con una topología, es natural pedir que las operaciones de grupo sean continuas, o si tenemos una medida $\mu$ en un grupo $G$, buscar que la medida de cualquier conjunto medible $A \subseteq G$ no cambie al operar sobre todos sus elementos, es decir, que

$$
\mu(A)=\mu(x A),
$$

para cada $x \in G$, donde $x A=\{x a \mid a \in A\}$. A esta última propiedad se le llama invarianza.

La medida de Haar es una medida definida sobre un grupo equipado con una topología tal que las tres estructuras - la algebraica, la topológica y la de medidason compatibles entre sí. Esta medida es invariante y, además, se puede determinar completamente a partir de la medida de los conjuntos compactos y de los conjuntos abiertos de la topología. El teorema de Haar garantiza la existencia de estas medidas cuando previamente se tiene una topología localmente compacta y un grupo que son compatibles entre sí. En la primera sección de este artículo presentamos brevemente este resultado, aunque sin demostración. El enfoque principal de este trabajo es el teorema de Haar inverso, que presentamos en la segunda sección. Este teorema nos permite construir topologías localmente compactas que son compatibles con el álgebra de grupo cuando previamente se tiene una medida invariante, transformando la medida invariante en una medida de Haar.

A lo largo de este trabajo, utilizaremos la palabra compatible de manera repetida para significar diferentes relaciones. Hablaremos de medidas de Radon como aquellas medidas que son compatibles con una topología, grupos topológicos como aquellos que tienen una topología compatible con las operaciones de grupo, y medidas invariantes como aquellas medidas que son compatibles con la estructura algebraica. Iremos especificando los detalles conforme requiramos los conceptos, así que el lector no debe preocuparse si no conoce algunos de ellos.

2010 Mathematics Subject Classification. 28C10, 43A05, 22D99.

Palabras clave. medida de Haar, medida invariante, topología de Weil. 


\section{UNA PROBADITA DEL TEOREMA DE HAAR}

2.1. Medidas de Radon (topología + medida). Estudiaremos exclusivamente espacios topológicos que son de Hausdorff (puntos distintos tienen vecindades ajenas) y localmente compactos (cada punto tiene una base local de vecindades compactas). Los espacios euclidianos $\mathbb{R}^{n}$, así como cualquier subconjunto cerrado o abierto de estos, son espacios de este tipo. Otro ejemplo se obtiene al tomar cualquier conjunto y equiparlo con la topología discreta.

Fijemos un espacio topológico de Hausdorff y localmente compacto $X$. El primer objetivo es especificar a qué nos referimos al pedir que la estructura topológica de $X$ sea compatible con las estructuras de medida que definiremos allí. Denotamos por $\mathcal{B}(X)$ a la $\sigma$-álgebra más pequeña que contiene a todos los conjuntos abiertos de $X$, y la llamamos la $\sigma$-álgebra de Borel. Es fácil notar que $\mathcal{B}(X)$ contiene a todos los conjuntos cerrados -pues son los complementos de los abiertos-, y por lo tanto $\mathcal{B}(X)$ contiene a los compactos de $X$, que siempre son cerrados en espacios de Hausdorff. Habiendo definido la $\sigma$-álgebra, nos adentramos a construir sobre ella medidas que también sean compatibles con la topología.

Sobre $X$, nos interesa integrar aquellas funciones continuas $f: X \rightarrow[0, \infty)$ que son cero fuera de un conjunto compacto. A las funciones en

$$
\mathcal{C}_{0}(X)=\left\{\begin{array}{l|l}
f: X \rightarrow[0, \infty) & \begin{array}{l}
f \text { es continua y existe un compacto } \\
S \subseteq X \text { tal que } f(x)=0 \text { si } x \notin S
\end{array}
\end{array}\right\}
$$

se les llama funciones continuas de soporte compacto. Por ejemplo, en $X=\mathbb{R}$ con la topología usual, las funciones $f_{1}, f_{2}, f_{3}: \mathbb{R} \rightarrow[0, \infty)$ dadas por

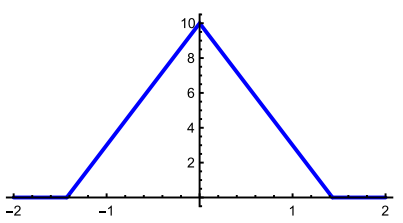

$f_{1}(x)=\operatorname{máx}\{0,10-7|x|\}$,

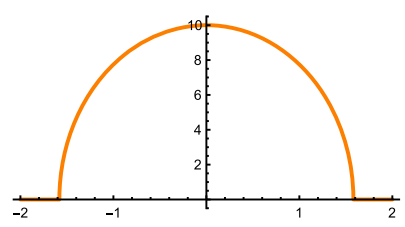

$f_{2}(x)=\sqrt{\operatorname{máx}\left\{0,100-40 x^{2}\right\}}$,

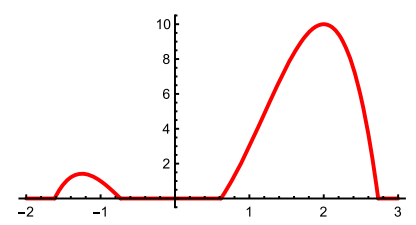

$f_{3}(x)=\operatorname{máx}\left\{0,5 x^{2}+x^{3}-x^{4}-2\right\}$

son continuas, y el conjunto compacto $S=[-2,3]$ satisface que $f_{1}(x)=f_{2}(x)=$ $f_{3}(x)=0$ si $x \notin S$, por lo que $f_{1}, f_{2}, f_{3} \in \mathcal{C}_{0}(\mathbb{R})$. Sin embargo, la función $f_{4}(x)=42$ no es un elemento de $\mathcal{C}_{0}(\mathbb{R})$, pues, aunque sí es continua, el único conjunto $S \subseteq \mathbb{R}$ tal que $f_{4}(x)=0$ si $x \notin S$ es $S=\mathbb{R}$, que no es compacto.

Es sencillo verificar que si $f, g \in \mathcal{C}_{0}(X)$ y $c \in[0, \infty)$, entonces $f+g \in \mathcal{C}_{0}(X)$ y $c f \in \mathcal{C}_{0}(X)$. Una integral en $\mathcal{C}_{0}(X)$ es un funcional $I: \mathcal{C}_{0}(X) \rightarrow[0, \infty)$ tal que $I(f+g)=I(f)+I(f)$ (es aditivo) y $\forall c \in[0, \infty): I(c f)=c I(f)$ (es homogéneo).

De nuevo, veamos un ejemplo en $X=\mathbb{R}$ con la topología euclidiana. La integral de Lebesgue $I(f)=\int_{-\infty}^{\infty} f(x) \mathrm{d} x$ para $f \in \mathcal{C}_{0}(\mathbb{R})$ es una integral en este sentido. En efecto, la aditividad, homogeneidad y el hecho de que $I(f) \geq 0$ deben ser obvios. Lo que tal vez no resulta evidente es por qué $I(f)<\infty$. Si $f \in \mathcal{C}_{0}(\mathbb{R})$, entonces existe $S \subseteq \mathbb{R}$ compacto tal que $f(x)=0$ si $x \notin S$. Como $S$ es compacto en $\mathbb{R}$, debe ser acotado, es decir, existe $N>0$ tal que $S \subseteq[-N, N]$. Además, siendo $f$ continua, existe $M=\operatorname{máx}\{f(x) \mid x \in[-N, N]\}$. Así,

$$
I(f)=\int_{-\infty}^{\infty} f(x) \mathrm{d} x=\int_{-N}^{N} f(x) \mathrm{d} x \leq \int_{-N}^{N} M \mathrm{~d} x=2 N M<\infty .
$$

Resulta que, en general, las integrales $I: \mathcal{C}_{0}(X) \rightarrow[0, \infty)$ siempre son las integrales de Lebesgue inducidas por una medida en $\mathcal{B}(X)$. Más aún, esta medida tiene ciertas propiedades que la hacen compatible con la topología.

Teorema 1 (de representación de Riesz-Markov-Kakutani (RMK)). Para toda integral $I: \mathcal{C}_{0}(X) \rightarrow[0, \infty)$ existe una única medida $\mu: \mathcal{B}(X) \rightarrow[0, \infty]$ tal 
que

$$
\int_{X} f(x) \mathrm{d} \mu(x)=I(f)
$$

para cada $f \in \mathcal{C}_{0}(X)$, y que satisface las siguientes condiciones:

(I) Todo compacto $C \subseteq X$ tiene medida finita $\mu(C)<\infty$. (Finitud local)

(II) Para todo $E \in \mathcal{B}(X)$ abierto o con $\mu(E)<\infty$,

$$
\mu(E)=\sup \{\mu(K) \mid K \subseteq E \text { compacto }\} . \quad \text { (Regularidad interior) }
$$

(III) Para todo $E \in \mathcal{B}(X)$,

$$
\mu(E)=\inf \{\mu(U) \mid U \supseteq E \text { abierto }\} . \quad \text { (Regularidad exterior) }
$$

Demostración. Ver [13, pp. 42-47].

A las medidas que satisfacen las propiedades (I) (II) y (III) se les conoce como medidas de Radon. Podemos determinar el valor de una medida de Radon a través de los conjuntos importantes topológicamente, es decir, aquellos abiertos y aquellos compactos. La medida de cualquier conjunto $E \in \mathcal{B}(X)$ es el ínfimo de las medidas de todos los conjuntos abiertos que lo contienen y, si $E$ es abierto o tiene medida finita, esta es el supremo de las medidas de todos los conjuntos compactos que contiene. Esto compatibiliza la estructura topológica con la de medida.

La medida de Lebesgue usual de $\mathbb{R}$ es justamente la medida correspondiente a la integral $I(f)=\int_{-\infty}^{\infty} f(x) \mathrm{d} x$ a través del teorema 1 , y por lo tanto es una medida de Radon. Otro ejemplo en cualquier espacio topologico localmente compacto y de Hausdorff $X$ se obtiene fijando $z \in X$ y definiendo la integral $I_{z}: \mathcal{B}(X) \rightarrow[0, \infty)$ por $I_{z}(f)=f(z)$ para cada $f \in \mathcal{C}_{0}(X)$. Por el teorema 1, existe una única medida de Radon $\delta_{z}: \mathcal{B}(X) \rightarrow[0, \infty]$ tal que $\int_{X} f(x) \mathrm{d} \delta_{z}(x)=f(z)$. Esta medida se llama la medida de Dirac centrada en $z$.

A continuación, veremos cómo se compatibiliza una estructura topológica con una algebraica.

2.2. Grupos topológicos (topología + álgebra). Nos interesan espacios topológicos que, además de topología, tengan una estructura de grupo. Pero debemos asegurarnos que el producto $(x, y) \mapsto x y$ y la inversión $x \mapsto x^{-1}$ respeten la topología, es decir, sean funciones continuas. Diremos que un grupo $G$ equipado con alguna topología de Hausdorff $\tau$ es un grupo topológico si las funciones $x \mapsto x^{-1}$ (de $G$ a $G$ ) y $(x, y) \mapsto x y($ de $G \times G$ a $G)$ son continuas, donde $G \times G$ es dotado con la topología producto usual.

Los números reales $\mathbb{R}$ con la topología euclidiana forman un grupo topológico, pues las funciones $x \mapsto-x$ y $(x, y) \mapsto x+y$ son continuas. Similarmente ocurre para el grupo de reales positivos $\mathbb{R}_{+}=(0, \infty)$, pero con el producto $x \mapsto 1 / x$ y $(x, y) \mapsto x y$. En general, cualquier grupo con la topología discreta - como el grupo de enteros $\mathbb{Z}$ con la topología heredada de $\mathbb{R}$ - es un grupo topológico, pues toda función cuyo dominio es discreto es continua. El grupo general lineal, de matrices invertibles de $n \times n$ con coeficientes en $\mathbb{C}$, es un grupo topológico si se le dota con la topología euclidiana de $\left(\mathbb{C}^{n}\right)^{n}$.

Es importante notar que la topología debe ser de Hausdorff para que un grupo sea un grupo topológico. Por ejemplo, si tomamos el grupo de números reales $\mathbb{R}$ con la topología indiscreta $\tau_{\text {ind }}=\{\emptyset, \mathbb{R}\}$, que no es de Hausdorff, entonces $\left(\mathbb{R}, \tau_{\text {ind }}\right)$ no es un grupo topológico, a pesar de que las funciones $x \mapsto-x$ y $(x, y) \mapsto x+y$ sí son continuas en este caso. Cabe mencionar, sin embargo, que algunos autores no imponen este requerimiento.

En los grupos topológicos, las operaciones de grupo preservan los conjuntos abiertos:

TeOrema 2. Sean $(G, \tau)$ un grupo topológico y $A \in \tau$. Entonces

$$
A^{-1}=\left\{a^{-1} \mid a \in A\right\}, \quad x A=\{x a \mid a \in A\} \quad y \quad A x=\{a x \mid a \in A\}
$$

son elementos de $\tau$ para todo $x \in G$. 
Demostración. Verifiquemos que $x A$ es un conjunto abierto. Los demás casos son similares. Definimos la función $f: G \rightarrow G$ por $f(y)=x^{-1} y$. Tenemos que

$$
f^{-1}(A)=\{y \in G \mid f(y) \in A\}=\left\{y \in G \mid x^{-1} y \in A\right\}=\{y \in G \mid y \in x A\}=x A .
$$

Es sencillo verificar que $y \mapsto(z, y)$ es una función continua para todo $z \in G$ y, como $G$ es un grupo topológico, $(x, y) \mapsto x y$ también lo es. Por lo tanto, la composición $f: y \mapsto\left(x^{-1}, y\right) \mapsto x^{-1} y$ es una función continua. Por definición de continuidad, $x A=f^{-1}(A)$ es un conjunto abierto.

Del teorema anterior se sigue que si $A$ es un elemento de $\mathcal{B}(G)$, entonces $x A, A x$ y $A^{-1}$ también son elementos de $\mathcal{B}(G)$. Resulta natural preguntarse si existen medidas de Radon que le asignen la misma medida a estos conjuntos, sin importar quién es $x$. Es decir, buscamos medidas en $G$ que sean compatibles tanto con el álgebra como con la topología.

2.3. La medida de Haar (topología + álgebra $\rightarrow$ medida). Sea $G$ un grupo topológico localmente compacto y $\mu: \mathcal{B}(G) \rightarrow[0, \infty]$ una medida. Decimos que $\mu$ es una medida invariante izquierda (derecha) si para todo $A \in \mathcal{B}(G)$ y $x \in G$, $\mu(x A)=\mu(A)(\mu(A x)=\mu(A))$. Una medida $\mu: \mathcal{B}(G) \rightarrow[0, \infty]$ es una medida de Haar izquierda (derecha) si $\mu$ es una medida de Radon no nula e invariante izquierda (derecha).

Dado que las medidas de Radon de $G$ están completamente determinadas por las integrales de las funciones $\mathcal{C}_{0}(G)$ (ver teorema 1), no es difícil probar (ver [10, 15.8, p. 193]) que si $\mu: \mathcal{B}(G) \rightarrow[0, \infty]$ es una medida de Radon, entonces $\mu$ es una medida de Haar izquierda si y solamente si para cada $f \in \mathcal{C}_{0}(G)$ y $y \in G$,

$$
\int_{G} f(y x) \mathrm{d} \mu(x)=\int_{G} f(x) \mathrm{d} \mu(x),
$$

y similarmente para el caso derecho.

La medida de Lebesgue usual en $\mathbb{R}$ es el primer ejemplo de una medida Haar izquierda (y derecha), pues, por el teorema de cambio de variable, tomando $u=y+x$, se tiene $\mathrm{d} u=\mathrm{d} x$, y luego

$$
\int_{-\infty}^{\infty} f(y+x) \mathrm{d} x=\int_{-\infty}^{\infty} f(x+y) \mathrm{d} x=\int_{-\infty}^{\infty} f(u) \mathrm{d} u=\int_{-\infty}^{\infty} f(x) \mathrm{d} x
$$

para cada $f \in \mathcal{C}_{0}(\mathbb{R})$ y $y \in \mathbb{R}$.

Otro ejemplo se obtiene al tomar el grupo de reales positivos $\mathbb{R}_{+}=(0, \infty)$ cuya operación es el producto. Utilizando el teorema de cambio de variable con $u=y x \mathrm{y}$ $\mathrm{d} u=y \mathrm{~d} x$ obtenemos

$$
\int_{0}^{\infty} f(y x) \mathrm{d} x=\int_{0}^{\infty} f(u) \frac{1}{y} \mathrm{~d} u=\frac{1}{y} \int_{0}^{\infty} f(x) \mathrm{d} x \neq \int_{0}^{\infty} f(x) \mathrm{d} x,
$$

si $y \neq 1$. Esto nos dice que la integral de Lebesgue usual $I(f)=\int_{0}^{\infty} f(x) \mathrm{d} x$ no corresponde a una medida de Haar izquierda en este grupo. Sin embargo, no todo está perdido, hay muchas otras medidas que podríamos definir. Consideremos la integral $H: \mathcal{B}\left(\mathbb{R}_{+}\right) \rightarrow[0, \infty)$ dada por $H(f)=\int_{0}^{\infty} \frac{1}{x} f(x) \mathrm{d} x$. Por el teorema 1 , existe una única medida de Radon $\mu: \mathcal{B}\left(\mathbb{R}_{+}\right) \rightarrow[0, \infty)$ tal que $\int_{0}^{\infty} \frac{1}{x} f(x) \mathrm{d} x=H(f)=\int_{\mathbb{R}_{+}} f(x) \mathrm{d} \mu(x)$ para cada $f \in \mathcal{C}_{0}\left(\mathbb{R}_{+}\right)$. La medida $\mu$ sí es una medida de Haar izquierda en $\mathbb{R}_{+}$pues, para cada $f \in \mathcal{C}_{0}\left(\mathbb{R}_{+}\right)$y $y \in \mathbb{R}_{+}$,

$\int_{\mathbb{R}_{+}} f(y x) \mathrm{d} \mu(x)=\int_{0}^{\infty} \frac{1}{x} f(y x) \mathrm{d} x=\int_{0}^{\infty} \frac{y}{u} f(u) \frac{1}{y} \mathrm{~d} u=\int_{0}^{\infty} \frac{1}{u} f(u) \mathrm{d} u=\int_{\mathbb{R}_{+}} f(x) \mathrm{d} \mu(x)$.

Un hecho con tintes milagrosos es que todos los grupos localmente compactos siempre poseen una medida de Haar, iy es única! -Bueno, casi única-. Este es el famoso teorema de Haar: 
TeOrema 3 (teorema de Haar). Sea $G$ un grupo topológico localmente compacto. Existe una medida de Haar izquierda (derecha) $\mu: \mathcal{B}(G) \rightarrow[0, \infty]$ que es única salvo por múltiplos reales positivos. Es decir, si tenemos otra medida de Haar izquierda (derecha) $\mu^{\prime}: \mathcal{B}(G) \rightarrow[0, \infty]$, existe $c>0$ tal que $\mu^{\prime}=c \mu$.

Este teorema, originalmente postulado en un caso particular por Haar en 1933 [8], ha sido motivo de miles de artículos y centenas de libros de texto. Cualquiera de sus posibles demostraciones es extensa, por lo que no lo probaremos aquí. Se pueden encontrar tratamientos muy accesibles en los libros [10, 7, 4, 5, basadas en la primera demostración dada por Weil [15] y en la demostración posterior de Cartan [3]. Una prueba menos conocida es la de Bredon [2] que se encuentra tratada con detalle en la referencia [12].

\section{El teorema de HaAr inverso}

El teorema de Haar permite construir una medida invariante a partir de una topología localmente compacta. El teorema de Haar inverso, originalmente demostrado por Weil en 1940 [15], ap. I] nos permite ir de regreso, es decir, construir una topología localmente compacta a partir de una medida invariante. En lo que resta del artículo, nos enfocaremos en demostrar este resultado. Seguiremos, en esencia, el camino original de Weil que se encuentra detallado en el libro de Halmos [9, cap. XII], aunque haremos algunas modificaciones sustanciales para evitar ciertos conceptos que han caído en desuso.

$$
\begin{array}{cccc}
\text { topología } & + \text { álgebra } & \text { teorema de Haar } & \text { medida } \\
\text { medida } & + \text { álgebra } & \stackrel{\text { teorema de Haar inverso }}{\longrightarrow} & \text { topología }
\end{array}
$$

3.1. Grupos medibles (medida + álgebra). Para poder definir una medida de Haar (una medida de Radon invariante) lo primero que fue necesario hacer fue armonizar la estructura topológica con la estructura algebraica. Esto lo hicimos pidiendo que las operaciones de grupo fueran continuas. Ahora queremos hablar de medidas invariantes sin una topología, por lo que debemos definir una $\sigma$-álgebra de tal manera que las operaciones de grupo respeten, ya no lo abierto, sino que lo medible. Recordemos que dados dos conjuntos $X$ y $Y$ con $\sigma$-álgebras $\mathcal{A}_{X}$ y $\mathcal{A}_{Y}$, respectivamente, una función $f: X \rightarrow Y$, es medible de $\mathcal{A}_{X}$ a $\mathcal{A}_{Y}$ si para cada $A \in \mathcal{A}_{Y}$, se tiene $f^{-1}(A) \in \mathcal{A}_{X}$

Diremos que un grupo $G$ equipado con una $\sigma$-álgebra $\mathcal{A}$ de subconjuntos de $G$ es un grupo medible si la función $x \mapsto x^{-1}$ es medible de $\mathcal{A}$ a $\mathcal{A}$ y la función $(x, y) \mapsto x y$ es medible de $\mathcal{A} \otimes \mathcal{A}$ a $\mathcal{A}$, donde $\mathcal{A} \otimes \mathcal{A}$ es la $\sigma$-álgebra producto, que es la $\sigma$-álgebra más pequeña que contiene al conjunto de rectángulos $\{A \times B \mid A, B \in \mathcal{A}\}$.

Como ejemplo, consideremos el grupo de números reales $\mathbb{R}$ con la suma y la $\sigma$-álgebra de Borel, $\mathcal{A}=\mathcal{B}(\mathbb{R})$. Veamos que $(\mathbb{R}, \mathcal{B}(\mathbb{R}))$ es un grupo medible. Como $\mathbb{R}$ es un grupo topológico, las funciones $x \mapsto-x$ y $(x, y) \mapsto x+y$ son continuas. Toda función continua es medible respecto a la $\sigma$-álgebra de Borel. Entonces $x \mapsto-x$ es medible de $\mathcal{B}(\mathbb{R})$ a $\mathcal{B}(\mathbb{R})$, y $(x, y) \mapsto x+y$ es medible de $\mathcal{B}(\mathbb{R} \times \mathbb{R})$ a $\mathcal{B}(\mathbb{R})$. Pero nosotros necesitamos que $(x, y) \mapsto x+y$ sea medible de $\mathcal{B}(\mathbb{R}) \otimes \mathcal{B}(\mathbb{R})$-no $\mathcal{B}(\mathbb{R} \times \mathbb{R})$ a $\mathcal{B}(\mathbb{R})$. Por suerte, en los números reales, ocurre que $\mathcal{B}(\mathbb{R} \times \mathbb{R})=\mathcal{B}(\mathbb{R}) \otimes \mathcal{B}(\mathbb{R})$. De hecho, es sencillo generalizar este argumento para cualquier grupo topológico segundo numerable $G$, pues en este caso siempre ocurre que $\mathcal{B}(G \times G)=\mathcal{B}(G) \otimes \mathcal{B}(G)$ (c.f. $[\underline{6}$, 4.1.7, p. 119]).

Si bien, en general, una $\sigma$-álgebra es una estructura muy diferente a una topología, resulta que muchos resultados sobre grupos topológicos se pueden calcar para grupos medibles. Por ejemplo, si en el teorema 2 sustituimos $\tau$ por $\mathcal{A}$, se obtiene el siguiente resultado. 
Teorema 4. Sean $(G, \mathcal{A})$ un grupo medible y $A \in \mathcal{A}$. Entonces

$$
A^{-1}=\left\{a^{-1} \mid a \in A\right\}, \quad x A=\{x a \mid a \in A\} \quad y \quad A x=\{a x \mid a \in A\}
$$

son elementos de $\mathcal{A}$ para todo $x \in G$.

Demostración. Calcar la demostración del teorema 2 pero sustituyendo

$\begin{array}{rlr}\text { conjunto abierto } & \text { por } & \text { conjunto medible, } \\ \text { función continua } & \text { por } & \text { función medible, } \\ \text { y grupo topológico } & \text { por } & \text { grupo medible. }\end{array}$

Sabiendo que las operaciones de grupo preservan la mensurabilidad, podemos volver a hablar de medidas invariantes, sin necesitar una topología. Una medida $\mu: \mathcal{A} \rightarrow[0, \infty]$ sobre un grupo medible $(G, \mathcal{A})$ es invariante izquierda (derecha) si $\mu(x A)=\mu(A)(\mu(A x)=\mu(A))$ para todo $A \in \mathcal{A}$ y $x \in G$.

Por ejemplo, en el grupo medible $(\mathbb{R}, \mathcal{B}(\mathbb{R}))$, la medida de Lebesgue es una medida invariante izquierda y derecha. Otro ejemplo sencillo es tomar un grupo finito $G=$ $\left\{x_{1}, \ldots, x_{n}\right\}$, con la $\sigma$-álgebra discreta $\mathcal{A}=\{A \mid A \subseteq G\}$. Es fácil verificar que $G$ es un grupo medible utilizando que $\mathcal{A} \otimes \mathcal{A}$ es la $\sigma$-álgebra discreta de $G \times G$. En este caso, la medida de conteo $\mu: G \rightarrow\{0,1, \ldots, n\}$, donde $\mu(A)$ es igual al número de elementos de $A$, es una medida invariante izquierda, pues si $A \subseteq G$, entonces $x A$ tiene el mismo número de elementos que $A$ para todo $x \in G$.

A partir de ahora solo trabajaremos con invarianza izquierda, pero cabe mencionar que todos los siguientes resultados se pueden reescribir para medidas invariantes derechas, cambiando el orden de todos los productos. Antes de empezar a topologizar los grupos medibles con medidas invariantes izquierdas, presentamos un par de resultados y notaciones importantes. Dado $X$ un conjunto y $\mathcal{A}$ una $\sigma$-álgebra, cuando digamos que $f: X \rightarrow[-\infty, \infty]$ es medible en $\mathcal{A}$, tomaremos a los conjuntos de Borel de los reales extendidos $[-\infty, \infty]$ como la $\sigma$-álgebra para el contradominio, que es la $\sigma$-álgebra generada por los conjuntos de la forma $[a, b)$ con $-\infty \leq a<b \leq \infty$.

El siguiente teorema nos permite calcular una especie de convolución de conjuntos con una medida invariante izquierda y $\sigma$-finita.

Teorema 5 (del promedio). Sean $(G, \mathcal{A})$ un grupo medible y $\mu: \mathcal{A} \rightarrow[0, \infty]$ una medida invariante izquierda y $\sigma$-finita. Para cualesquiera $A, B \in \mathcal{A}$, la función $x \mapsto \mu\left(x^{-1} A \cap B\right)$ es medible $y$

$$
\int_{G} \mu\left(x^{-1} A \cap B\right) \mathrm{d} \mu(x)=\mu(A) \mu\left(B^{-1}\right) .
$$

Demostración. Ver [9, Sec. 59, teo. F, p. 261] o [12, 3.2.11].

Con el teorema del promedio podemos mostrar que si bien las inversiones o traslaciones derechas pueden no preservar la medida de un conjunto bajo una medida invariante izquierda y $\sigma$-finita, estas operaciones sí preservan su positividad.

Corolario 6. Sean $(G, \mathcal{A})$ un grupo medible y $\mu: \mathcal{A} \rightarrow[0, \infty]$ una medida invariante izquierda y $\sigma$-finita. Si $A \in \mathcal{A}$ satisface $\mu(A)>0$, entonces $\mu\left(A^{-1}\right)>0$ y $\mu(A a)>0$ para todo $a \in G$.

Demostración. El teorema del promedio nos dice que

$$
\int_{G} \mu\left(x^{-1} A \cap A^{-1}\right) \mathrm{d} \mu(x)=\mu(A)^{2}>0 .
$$

Por lo tanto, existe $x \in G$ tal que $B=x^{-1} A \cap A^{-1}$ tiene medida positiva, y entonces debe ocurrir que $\mu\left(A^{-1}\right)>0$. Si repetimos el mismo argumento para el conjunto $a^{-1} B$ con medida $\mu\left(a^{-1} B\right)=\mu(B)>0$, debe existir $y \in G$ tal que $C=y^{-1} a^{-1} B \cap\left(a^{-1} B\right)^{-1}$ tiene medida positiva. Dado que $C \subseteq B^{-1} a \subseteq A a$, concluimos que $\mu(A a)>0$. 
El hecho de que la familia de conjuntos de medida positiva se mantenga invariante ante cualquiera de las operaciones de grupo resultará muy importante. Lo que haremos en la siguiente sección es postular como abiertos precisamente a ciertos conjuntos de medida positiva y con ello construir una topología para el grupo medible.

3.2. La topología de Weil (medida + álgebra $\rightarrow$ topología). Recordemos que es posible construir topologías a partir de bases locales de vecindades abiertas. Pidiendo ciertas condiciones sobre una familia de subconjuntos de un grupo, podemos garantizar que existe una topología donde este es un grupo topológico que tiene a esa familia como una base local de vecindades abiertas.

TeOrema 7. Sea $G$ un grupo y consideremos una familia no vacía $\mathcal{V}$ de subconjuntos de $G$ tal que:

(a) Para todo $V \in \mathcal{V}, e \in V$ (e es el elemento neutro de $G$ )

(b) Dados $U, V \in \mathcal{V}$, existe $W \in \mathcal{V}$ tal que $W \subseteq V \cap U$.

(c) Para cada $U \in \mathcal{V}$ y $x \in U$, existe $V \in \mathcal{V}$ con $x V \subseteq U$.

(d) Para cada $U \in \mathcal{V}$ existe $V \in \mathcal{V}$ tal que $V V^{-1} \subseteq U$.

(e) Dados $U \in \mathcal{V}$ y $x \in G$, existe $V \in \mathcal{V}$ tal que $V \subseteq x U x^{-1}$.

(f) Si $x \in G$ y $x \neq e$, entonces existe $V \in \mathcal{V}$, tal que $x \notin V$.

Entonces la familia

$$
\mathcal{V}(x)=\{x V \mid V \in \mathcal{V}\}
$$

es una base local de vecindades abiertas para cada $x \in G$ en una única topología con la cual $G$ es un grupo topológico.

Demostración. Ver [12, 1.1.12] o [1, 1.3.12, p. 22].

Para construir una topología en un grupo medible que lo convierta en un grupo topológico, podemos construir una familia $\mathcal{V}$ como la del teorema 7. Utilicemos el siguiente ejemplo para motivar el procedimiento que desarrollaremos más adelante.

Consideremos el grupo medible $\mathbb{R}$ con la suma y la $\sigma$-álgebra de Borel. Sobre él tenemos la medida invariante izquierda $\mu: \mathcal{B}(\mathbb{R}) \rightarrow[0, \infty]$ dada por la medida usual de Lebesgue. Ya sabemos que la topología usual hace que $\mathbb{R}$ sea un grupo topológico. Veamos si podemos construir una base local de vecindades abiertas para esta topología utilizando solo la medida $\mu$. Lo primero que se debería venir a la mente cuando uno dice base local de vecindades abiertas para $\mathbb{R}$ es la familia de intervalos (bolas) abiertos. Consideremos

$$
\mathcal{V}=\{B(\varepsilon, 0) \mid \varepsilon>0\}, \quad \text { donde } \quad B(\varepsilon, x)=\{y \in \mathbb{R}|| y-x \mid<\varepsilon\}=(x-\varepsilon, x+\varepsilon) .
$$

Es sencillo verificar que la familia $\mathcal{V}$ satisface las condiciones del teorema 7 y entonces la familia

$$
\mathcal{V}(x)=\{x+V \mid V \in \mathcal{V}\}=\{B(\varepsilon, x) \mid \varepsilon>0\}
$$

es una base local de vecindades abiertas para cada $x \in \mathbb{R}$ en una única topología con la cual $\mathbb{R}$ es un grupo topológico, que es la topología usual de $\mathbb{R}$.

Observemos que para construir las bolas $B(\varepsilon, x)$ utilizamos el valor absoluto $|\cdot|$, que es una norma en $\mathbb{R}$. ¿Habrá forma de calcular esta norma utilizando la medida de Lebesgue $\mu$ ? Es decir, dado $x \in \mathbb{R}$, ¿podemos calcular $|x|$ utilizando solo $\mu$ ?

Si tomamos $A=(-M, M) \in \mathcal{B}(\mathbb{R})$ donde $M>0$, obtenemos $x+A=(x-M, x+M)$. Si $|x| \geq 2 M$, entonces $A \cap(x+A)=\emptyset$, y por lo tanto $\mu(A \cap(x+A))=0$. Pero si $|x|<2 M$, el siguiente esquema debería convencer al lector de que $\mu(A \cap(x+A))+|x|=$ $\mu(A)$.

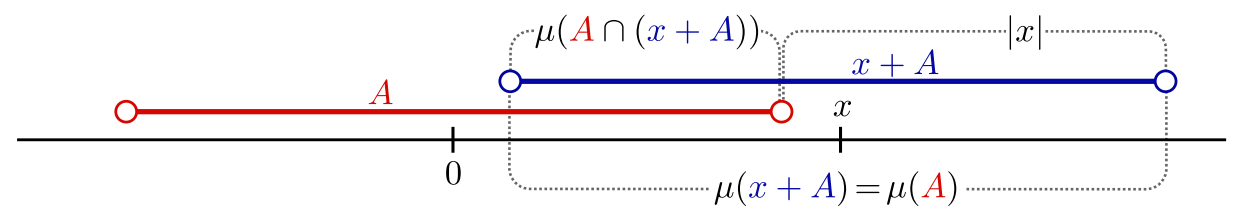


Por lo tanto,

$$
|x|=\mu(A)-\mu(A \cap(x+A)) \quad(\text { si }|x|<2 M) .
$$

Regresemos al caso general. Fijemos un grupo medible $(G, \mathcal{A})$ y una medida invariante izquierda $\mu: \mathcal{A} \rightarrow[0, \infty]$ cualesquiera. Inspirados por el ejemplo anterior, definimos

$$
\|x\|_{\mu}^{A}=\mu(A)-\mu(A \cap x A)
$$

para cada $A \in \mathcal{A}$ con $0<\mu(A)<\infty$ y $x \in G$.

Lema 8. Para cualesquiera $x, y \in G$ y $A \in \mathcal{A}$ con $0<\mu(A)<\infty$ se satisfacen las siguientes condiciones:

(I) $\left\|\left|\|\mid\|_{\mu}^{A}=0\right.\right.$.

(II) $\|x\|\left\|_{\mu}^{A}=\right\| x^{-1} \|_{\mu}^{A} \cdot$ (Simetría)

(III) \|\|$x y\|\|_{\mu}^{A} \leq\|\| x\left\|_{\mu}^{A}+\right\| y\|\|_{\mu}^{A}$. (Desigualdad del triángulo)

Demostración. Verifiquemos cada inciso por separado.

(I) Directamente de la definición

$$
\|e\|_{\mu}^{A}=\mu(A)-\mu(A \cap e A)=\mu(A)-\mu(A \cap A)=\mu(A)-\mu(A)=0 .
$$

(II) Como $\mu$ es invariante izquierda,

$$
\mu(A \cap x A)=\mu\left(x^{-1}(A \cap x A)\right)=\mu\left(x^{-1} A \cap x^{-1} x A\right)=\mu\left(x^{-1} A \cap A\right),
$$

y entonces

$$
\|x \mid\|_{\mu}^{A}=\mu(A)-\mu(A \cap x A)=\mu(A)-\mu\left(x^{-1} A \cap A\right)=\left\|x^{-1}\right\|_{\mu}^{A} .
$$

(III) Primero notemos que $A \supseteq A \cap\left(x^{-1} A \cup y A\right)=\left(A \cap x^{-1} A\right) \cup(A \cap y A)$, y entonces

$$
\begin{aligned}
\mu(A) & \geq \mu\left(\left(A \cap x^{-1} A\right) \cup(A \cap y A)\right) \\
& =\mu\left(A \cap x^{-1} A\right)+\mu(A \cap y A)-\mu\left(A \cap x^{-1} A \cap y A\right) .
\end{aligned}
$$

Ahora, de nuevo por la invarianza izquierda de $\mu$ se tiene

$$
\mu(A \cap x y A)=\mu\left(x^{-1} A \cap y A\right),
$$

y como $A \cap x^{-1} A \cap y A \subseteq x^{-1} A \cap y A$, obtenemos

$$
\begin{aligned}
\|x y\|_{\mu}^{A} & =\mu(A)-\mu\left(x^{-1} A \cap y A\right) \leq \mu(A)-\mu\left(A \cap x^{-1} A \cap y A\right) \\
& \leq \mu(A)-\mu\left(A \cap x^{-1} A\right)+\mu(A)-\mu(A \cap y A)=\left\|x^{-1}\right\|_{\mu}^{A}+\|y\|_{\mu}^{A} .
\end{aligned}
$$

Por la simetría, $\left\|x^{-1}\right\|_{\mu}^{A}=\|x\|_{\mu}^{A}$, dándonos la desigualdad deseada.

Podríamos estar tentados a llamar a las funciones $\|\cdot \cdot\|_{\mu}^{A}$ normas de grupo, en similitud a las normas en espacios vectoriales. Sin embargo, las normas en espacios vectoriales cumplen la propiedad de que $\|v\|=0$ si y solamente si $v$ es el vector cero. Aquí, puede pasar que $\|x\|_{\mu}^{A}=0$ aunque $x \neq e$. Por ejemplo, en un grupo de medida finita $0<\mu(G)<\infty$ (más adelante construimos un ejemplo explícito de este tipo), se tiene $\|x\|_{\mu}^{G}=\mu(G)-\mu(G)=0$ para todo $x \in G$. Por ello, a las funciones $\|\cdot\| \cdot \|$ que cumplen las propiedades del lema 8 se les llama seudonormas de grupo 11 .

Con las seudonormas $\|\cdot \mid\|_{\mu}^{A}$ definiremos una familia de bolas, de la manera usual. Para cada $\varepsilon>0$ y $A \in \mathcal{A}$ con $\varepsilon<\mu(A)<\infty$, escribimos

$$
B_{\mu}(\varepsilon, A)=\left\{x \in G \mid\|x\|_{\mu}^{A}<\varepsilon\right\} .
$$

Pedimos que $\varepsilon<\mu(A)$ porque es el caso interesante. Cuando $\varepsilon>\mu(A)$, se tiene $\|x\|_{\mu}^{A} \leq \mu(A)<\varepsilon \sin$ importar quién es $x \in G$. Denotamos por $\mathcal{V}_{\mu}$ a la familia de todas las bolas $B_{\mu}(\varepsilon, A)$, es decir,

$$
\mathcal{V}_{\mu}=\left\{B_{\mu}(\varepsilon, A) \mid A \in \mathcal{A}, \quad \varepsilon>0 \quad \text { y } \quad \varepsilon<\mu(A)<\infty\right\} .
$$

Notemos para todo $B_{\mu}(\varepsilon, A) \in \mathcal{V}_{\mu}$, se tiene $B_{\mu}(\varepsilon, A) \in \mathcal{A}$, pues la función

$$
f(x)=\mu(x A \cap A)=\mu\left(x^{-1} A \cap A\right)
$$


es una función medible (ver el teorema 5 y $B_{\mu}(\varepsilon, A)=f^{-1}([0, \mu(A)-\varepsilon)$ ). También notemos que por la simetría de $\|\cdot\|_{\mu}^{A}$, se tiene $B_{\mu}(\varepsilon, A)=B_{\mu}(\varepsilon, A)^{-1}$. Los conjuntos $B_{\mu}(\varepsilon, A)$ en $\mathcal{V}_{\mu}$ serán vecindades para $e$ en la topología que buscamos.

TeOrema 9. La familia $\mathcal{V}_{\mu}$ satisface los incisos (a), (c) y (d) del teorema 7 .

Demostración. Estos incisos se deducen a partir de las propiedades del lema 8. Sea $B_{\mu}(\varepsilon, A) \in \mathcal{V}_{\mu}$.

(a) Como $\|e\| \|_{\mu}^{A}=0<\varepsilon$, tenemos $e \in B_{\mu}(\varepsilon, A)$.

(c) Para $x \in B_{\mu}(\varepsilon, A)$, definimos $\delta=\varepsilon-\|x\|_{\mu}^{A}>0$. Por la definición de $\mathcal{V}_{\mu}$, se tiene $\mu(A)>\varepsilon$, y entonces $\mu(A)>\delta$. Por lo tanto, podemos considerar la bola $B_{\mu}(\delta, A) \in \mathcal{V}_{\mu}$. Si $y \in B_{\mu}(\delta, A)$,

$$
\|x y\|_{\mu}^{A} \leq\|\| x\left\|_{\mu}^{A}+\right\| y\left\|_{\mu}^{A}<\right\| x \|_{\mu}^{A}+\delta=\varepsilon,
$$

y así $x y \in B_{\mu}(\varepsilon, A)$. Por lo tanto, $x B_{\mu}(\delta, A) \subseteq B_{\mu}(\varepsilon, A)$.

(d) Se tiene $B_{\mu}(\varepsilon / 2, A) B_{\mu}(\varepsilon / 2, A)^{-1} \subseteq B_{\mu}(\varepsilon, A)$, pues si

$$
x \in B_{\mu}(\varepsilon / 2, A) B_{\mu}(\varepsilon / 2, A)^{-1},
$$

entonces $x=a b^{-1}$ para ciertos $a, b \in B_{\mu}(\varepsilon / 2, A)$. Luego,

$$
\||x|\|_{\mu}^{A} \leq\|a \mid\|_{\mu}^{A}+\|b\|_{\mu}^{A}<\varepsilon / 2+\varepsilon / 2=\varepsilon,
$$

y por lo tanto $x \in B_{\mu}(\varepsilon, A)$.

Para demostrar los demás incisos, necesitaremos hacer un poco más de trabajo, e imponer algunas condiciones adicionales sobre la medida invariante. Regresemos al ejemplo de los números reales.

Consideremos la medida invariante izquierda de Lebesgue $\mu: \mathcal{B}(\mathbb{R}) \rightarrow[0, \infty]$. Tomando $M>0$ y $A=(-M, M) \in \mathcal{B}(\mathbb{R})$, ya vimos que $\||x|\|_{\mu}^{A}=\operatorname{mín}\{|x|, 2 M\}$. Por lo tanto, si $0<\varepsilon<\mu(A)=2 M$,

$$
B_{\mu}(\varepsilon, A)=\left\{x \in \mathbb{R}\left|\|\mid\|_{\mu}^{A}<\varepsilon\right\}=\{x \in \mathbb{R}|| x \mid<\varepsilon\}=(-\varepsilon, \varepsilon) .\right.
$$

Escribamos $C-C=\{x-y \mid x, y \in C\}$ para cualquier $C \subseteq \mathbb{R}$. Entonces

$$
A-A=\{x-y \mid-M<x<M,-M<y<M\}=(-2 M, 2 M) \supseteq(-\varepsilon, \varepsilon)=B_{\mu}(\varepsilon, A) \text {. }
$$

Por otro lado, si definimos $E=(-\varepsilon / 4, \varepsilon / 4) \in \mathcal{B}(\mathbb{R})$, tenemos que

$$
E-E=\{x-y \mid x, y \in E\}=(-\varepsilon / 2, \varepsilon / 2) \subseteq(-\varepsilon, \varepsilon)=B_{\mu}(\varepsilon, A) .
$$

Es decir, hemos visto que

$$
E-E \subseteq B_{\mu}(\varepsilon, A) \subseteq A-A
$$

donde $E \in \mathcal{B}(\mathbb{R})$ satisface $0<\mu(E)<\infty$. Resulta que esto siempre ocurre, independientemente del grupo y de quién es $A$, siempre y cuando la medida $\mu$ sea $\sigma$-finita.

En un grupo $G$ cualquiera, dados $A, B \subseteq G$, escribimos

$$
A B=\{a b \mid a \in A, b \in B\} \quad \text { y } \quad A^{-1}=\left\{a^{-1} \mid a \in A\right\},
$$

y así, en particular, el conjunto

$$
A A^{-1}=\left\{a_{1} a_{2}^{-1} \mid a_{1}, a_{2} \in A\right\}
$$

generaliza la notación $A-A$ que teníamos para $\mathbb{R}$. El resultado que encontramos en el ejemplo anterior se generaliza de la siguiente manera.

Lema 10. Sean $(G, \mathcal{A})$ un grupo medible y $\mu: \mathcal{A} \rightarrow[0, \infty]$ una medida invariante izquierda y $\sigma$-finita. Si $B_{\mu}(\varepsilon, A) \in \mathcal{V}_{\mu}$, entonces:

(I) $B_{\mu}(\varepsilon, A) \subseteq A A^{-1}$.

(II) Dado $F \in \mathcal{A}$ con $\mu(F)>0$, existe $E \subseteq F$ medible con $0<\mu(E)<\infty$ y tal que

$$
E E^{-1} \subseteq B_{\mu}(\varepsilon, A) \text {. }
$$

$\mathrm{Al}$ inciso (II) se le conoce como el lema de fragmentación de Weil. 


\section{Demostración.}

(I) Sea $x \in B_{\mu}(\varepsilon, A)$. Por definición, $0<\mu(A)-\varepsilon<\mu(A \cap x A)$ y entonces $A \cap x A \neq \emptyset$. Luego, existe $a \in A \cap x A$, y por lo tanto $a=x a^{\prime}$ para algún $a^{\prime} \in A$. Así, $x=a a^{\prime-1} \in A A^{-1}$.

(II) Ver [9, Sec. 62, teo. A, p. 270] o [12, 3.3.12].

El lema 10 nos permite demostrar dos más de las propiedades que necesitamos para poder aplicar el teorema 7

Teorema 11. Sea $(G, \mathcal{A})$ un grupo medible y $\mu: \mathcal{A} \rightarrow[0, \infty]$ una medida invariante izquierda y $\sigma$-finita. La familia $\mathcal{V}_{\mu}$ satisface los incisos (b) y (e) del teorema 7 .

Demostración. El lema 10 nos dice que todo conjunto en $\mathcal{V}_{\mu}$ contiene a (y está contenido en) un conjunto de la familia

$$
\mathcal{N}=\left\{A A^{-1} \mid A \in \mathcal{A}, \quad 0<\mu(A)<\infty\right\} .
$$

Por lo tanto, basta que veamos que la familia $\mathcal{N}$ satisface los incisos (b) y (e)

(b) Sean $U=A A^{-1} \in \mathcal{N}$ y $V=B B^{-1} \in \mathcal{N}$. Buscamos $W=C C^{-1} \in \mathcal{N}$ con $W \subseteq U \cap V$. Como $\mu(A)>0$, por el corolario 6, $\mu\left(A^{-1}\right)>0$. Luego $\mu\left(A^{-1}\right) \mu(B)>0$ y, por el teorema del promedio (teorema 5,

$$
\int_{G} \mu\left(x^{-1} A^{-1} \cap B^{-1}\right) \mathrm{d} \mu(x)>0 .
$$

Entonces debe existir algún $x \in G$ tal que $\mu\left(x^{-1} A^{-1} \cap B^{-1}\right)>0$. Tomando $D=A x \cap B$, tenemos $D^{-1}=x^{-1} A^{-1} \cap B^{-1}$, y entonces $\mu(D)>0$. Como $\mu$ es $\sigma$-finita, existe $C \subseteq D$ medible con $0<\mu(C)<\infty$. Así, $C C^{-1} \in \mathcal{N}$ y además, dado que $D \subseteq B$, tenemos $D D^{-1} \subseteq B B^{-1}$ y, dado que $D x^{-1} \subseteq A$, tenemos

$$
D D^{-1}=D x^{-1} x D^{-1}=D x^{-1}\left(D x^{-1}\right)^{-1} \subseteq A A^{-1} .
$$

Por lo tanto,

$$
W=C C^{-1} \subseteq D D^{-1} \subseteq A A^{-1} \cap B B^{-1}=U \cap V .
$$

(e) Si $U=A A^{-1} \in \mathcal{N}$ y $x \in G$ entonces tomando $V=(x A)(x A)^{-1} \in \mathcal{N}$, tenemos

$$
V=x A A^{-1} x^{-1}=x U x^{-1} .
$$

Resta verificar que la familia $\mathcal{V}_{\mu}$ satisface el inciso(f) del teorema 7. Este inciso es el que nos garantiza que la topología generada por el teorema 7 sea de Hausdorff, como requerimos en la definición de grupo topológico. Desafortunadamente, no cualquier medida invariante y $\sigma$-finita hace que $\mathcal{V}_{\mu}$ satisfaga esta propiedad.

Un ejemplo sencillo es tomar el grupo medible $\left(\mathbb{R}, \mathcal{A}_{\text {ind }}\right)$, con la $\sigma$-álgebra indiscreta $\mathcal{A}_{\text {ind }}=\{\emptyset, \mathbb{R}\}$ y la medida invariante izquierda $\mu_{\text {ind }}: \mathcal{A}_{\text {ind }} \rightarrow\{0,1\}$ dada por $\mu_{\text {ind }}(\emptyset)=0$ y $\mu_{\text {ind }}(\mathbb{R})=1$. Para todo $x \in \mathbb{R}$ se tiene

$$
\|x\|_{\mu_{\text {ind }}}^{\mathbb{R}}=\mu_{\text {ind }}(\mathbb{R})-\mu_{\text {ind }}(\mathbb{R} \cap(x+\mathbb{R}))=\mu_{\text {ind }}(\mathbb{R})-\mu_{\text {ind }}(\mathbb{R})=1-1=0 .
$$

Por lo tanto, si $0<\varepsilon<\mu_{\text {ind }}(\mathbb{R})=1$, se tiene $B_{\mu_{\text {ind }}}(\varepsilon, \mathbb{R})=\mathbb{R}$, y luego $\mathcal{V}_{\mu_{\text {ind }}}=\{\mathbb{R}\}$. Si $x \neq 0$, entonces $x \in V$ para todo $V \in \mathcal{V}_{\mu_{\text {ind }}}=\{\mathbb{R}\}$, contrario al inciso (f) del teorema 7. Sin embargo, evidentemente, la única topología en la cual la familia $\{\mathbb{R}\}$ es una base local de vecindades abiertas es la topología indiscreta, que precisamente no es de Hausdorff.

La solución a este obstáculo es directamente imponer que se satisfaga el inciso (f) del teorema 7. Diremos que una medida invariante izquierda $\mu: \mathcal{A} \rightarrow[0, \infty]$ en un grupo medible $(G, \mathcal{A})$ es separadora si $\mathcal{V}_{\mu}$ satisface el inciso (f) del teorema 7 . Es sencillo verificar que esto es equivalente a pedir que para para cada $x \in G$ con $x \neq e$, exista $A \in \mathcal{A}$ con $\mu(A \cap x A)<\mu(A)<\infty$. Esta propiedad en realidad no es muy restrictiva; imponerla solo nos permite excluir ciertas medidas triviales, como $\mu_{\text {ind }}$. Por ejemplo, la medida de Lebesgue $\mu: \mathcal{B}(\mathbb{R}) \rightarrow[0, \infty]$ es separadora, pues para cada 
$x \in \mathbb{R}$ con $x \neq 0$, tomando $A=(-|x| / 2,|x| / 2)$ obtenemos $A \cap(x+A)=\emptyset$ y entonces $\mu(A \cap(x+A))=0<|x|=\mu(A)$.

Si una medida es invariante izquierda, $\sigma$-finita, separadora y no nula diremos que es una medida de Weil. Hemos demostrado que si $(G, \mathcal{A})$ es un grupo medible y $\mu: \mathcal{A} \rightarrow[0, \infty]$ una medida de Weil, entonces se satisfacen todos los incisos del teorema 7. Este teorema requiere, adicionalmente, que la familia $\mathcal{V}_{\mu}$ sea no vacía. Por ello pedimos que la medida de Weil $\mu$ sea no nula. Esta condición, junto con la $\sigma$-finitud, garantiza la existencia de algún $A \in \mathcal{A}$ con $0<\mu(A)<\infty$, y en consecuencia $B_{\mu}(\varepsilon, A) \in \mathcal{V}_{\mu}$ para todo $\varepsilon \in(0, \mu(A))$. Así, podemos aplicar el teorema 7 , dándonos una única topología $\tau_{\mu}$ con base local de vecindades abiertas

$$
\left\{x V \mid V \in \mathcal{V}_{\mu}\right\} \quad\left(\mathcal{V}_{\mu}=\left\{B_{\mu}(\varepsilon, A) \mid A \in \mathcal{A}, 0<\varepsilon<\mu(A)<\infty\right\}\right) .
$$

Esta topología se llama la topología de Weil inducida por $\mu$.

Recordemos que las topologías en las cuales existía una única medida de Haar (salvo múltiplos reales positivos) eran aquellas localmente compactas. La topología de Weil es casi localmente compacta, es decir, equipado con ella, el grupo medible es un subgrupo denso de un grupo topológico localmente compacto. Para demostrar este hecho, introducimos el siguiente concepto.

Dado un grupo topológico $G$, decimos que $V \subseteq G$ es totalmente acotado si para cualquier vecindad $U$ de $e$, existen $x_{1}, \ldots, x_{n} \in G$ tales que

$$
V \subseteq \bigcup_{i=1}^{n} x_{i} U .
$$

Esta definición generaliza aquella de los espacios métricos, donde se dice que un conjunto es totalmente acotado si se puede cubrir con una cantidad finita de bolas de cualquier radio.

Resulta que un grupo topológico es un subgrupo denso de un grupo localmente compacto si existe una vecindad $V$ de $e$ que sea totalmente acotada. Este hecho fue originalmente demostrado por Weil en [14, cap. 3]. Se puede consultar la demostración en la referencia [1, p. 398]. Veremos que la topología inducida por una medida de Weil satisface siempre esta propiedad.

Lema 12. Sean $(G, \mathcal{A})$ un grupo medible y $\mu: \mathcal{A} \rightarrow[0, \infty]$ una medida de Weil. Existe una vecindad $V \in \mathcal{V}_{\mu}$ que es totalmente acotada en la topología de Weil inducida por $\mu$. Más aún, si $E \in \mathcal{A}$ es totalmente acotado en esta topología, entonces $\mu(E)<\infty$.

Demostración. Lo primero que haremos es construir $W \in \mathcal{V}_{\mu}$ de medida finita. Como $\mu$ es $\sigma$-finita y no nula, existe $A \in \mathcal{A}$ tal que $0<\mu(A)<\infty$. Por el corolario 6 , $\mu\left(A^{-1}\right)>0$, y por la $\sigma$-finitud podemos suponer, sin pérdida de generalidad, que $\mu\left(A^{-1}\right)<\infty$. Fijemos un $\varepsilon \in(0, \mu(A))$ y sea $W=B_{\mu}(\varepsilon, A) \in \mathcal{V}_{\mu}$. Del lema 10 deducimos que $W \subseteq A A^{-1}$ y, por definición, para cualquier $x \in W$ se satisface que $\mu(A)-\mu\left(x^{-1} A \cap A\right)=\|\| x \|_{\mu}^{A}<\varepsilon$. Despejando, obtenemos que

$$
\frac{\mu\left(x^{-1} A \cap A\right)}{\mu(A)-\varepsilon}>1
$$

para cualquier $x \in W$. Del teorema del promedio (teorema 5) se sigue que

$$
\begin{aligned}
\mu(W) & =\int_{W} 1 \mathrm{~d} \mu(x) \leq \int_{W} \frac{\mu\left(x^{-1} A \cap A\right)}{\mu(A)-\varepsilon} \mathrm{d} \mu(x) \leq \int_{G} \frac{\mu\left(x^{-1} A \cap A\right)}{\mu(A)-\varepsilon} \mathrm{d} \mu(x) \\
& =\frac{\mu(A) \mu\left(A^{-1}\right)}{\mu(A)-\varepsilon}<\infty .
\end{aligned}
$$

Así, hemos obtenido una vecindad $W \in \mathcal{V}_{\mu}$ de medida finita. Notemos que esto demuestra la segunda parte del enunciado, pues si $E \in \mathcal{A}$ es totalmente acotado, se puede cubrir con una cantidad finita de conjuntos de la forma $x W(x \in G)$, que tienen medida finita. 
Veamos que en efecto existe una vecindad $V \in \mathcal{V}_{\mu}$ totalmente acotada. Como en la demostración del teorema 9 inciso (d), podemos construir $V \in \mathcal{V}_{\mu}$ tal que $V V^{-1} \subseteq W$. Demostraremos que $V$ es totalmente acotada. Por contradicción, supongamos que existe una vecindad $U$ de $e$ tal que $V \nsubseteq \bigcup_{i=1}^{n} x_{i} U$ para cualesquiera $x_{1}, \ldots, x_{n} \in G$. Como $\mathcal{V}_{\mu}$ es una base local para $e$, podemos suponer que $U \in \mathcal{V}_{\mu}$.

Por medio de la propiedad de $U$ construyamos una sucesión de puntos en $V$. Tomemos $x_{1}=e \in V$. Como $V \nsubseteq x_{1} U$, existe $x_{2} \in V$ con $x_{2} \notin x_{1} U$. De nuevo, como $V \nsubseteq x_{1} U \cup x_{2} U$ existe $x_{3} \in V$ con $x_{3} \notin x_{1} U \cup x_{2} U$. Siguiendo de esta manera, para cada $n \in \mathbb{N}$, dado que $V \nsubseteq \bigcup_{i=1}^{n} x_{i} U$, podemos tomar $x_{n+1} \in V$ con $x_{n+1} \notin \bigcup_{i=1}^{n} x_{i} U$.

Por el lema 10 (II) existe $F \in \mathcal{A}$ con $0<\mu(F)<\infty, F \subseteq V^{-1}$ y $F F^{-1} \subseteq U$. Si $n>m$, entonces $x_{n} \notin x_{m} U \supseteq x_{m} F F^{-1}$, es decir, no existe $z \in F$ tal que $x_{n} z \in x_{m} F$, por lo que $x_{n} F \cap x_{m} F=\emptyset$. Con lo anterior, hemos construido una familia de conjuntos ajenos con medida finita y positiva $\left\{x_{n} F \mid n \in \mathbb{N}\right\}$. Además, se tiene

$$
x_{n} F \subseteq x_{n} V^{-1} \subseteq V V^{-1} \subseteq W
$$

para todo $n \in \mathbb{N}$, por lo que

$$
\mu(W) \geq \mu\left(\bigcup_{n \in \mathbb{N}} x_{n} F\right)=\sum_{n=1}^{\infty} \mu\left(x_{n} F\right)=\sum_{n=1}^{\infty} \mu(F)=\infty,
$$

lo cual contradice que $\mu(W)<\infty$. Esta contradicción viene de suponer que la vecindad $V$ no es totalmente acotada, por lo que debe serlo.

Hemos llegado, al fin, al esperado teorema de Haar inverso. A partir de una medida de Weil pudimos construir una topología que es densamente encajable en un grupo localmente compacto. Por el teorema de Haar, este grupo localmente compacto debe tener una medida de Haar izquierda. Lo que resulta fantástico es que esta medida de Haar está completamente determinada por la medida de Weil con la que empezamos. Esto nos dice que no solo ocurre que las topologías localmente compactas producen medidas de Radon invariantes, sino que además las medidas invariantes en cualquier grupo medible son, en esencia, medidas de Radon en una topología localmente compacta.

Teorema 13 (de Haar inverso de Weil). Consideremos un grupo medible $(G, \mathcal{A})$ y una medida de Weil $\mu: \mathcal{A} \rightarrow[0, \infty]$. Con la topología de Weil inducida por $\mu, G$ es un subgrupo denso de un grupo topológico localmente compacto $\widehat{G}$. Más aún, si $f \in \mathcal{C}_{0}(\widehat{G})$, la restricción $\left.f\right|_{G}$ es medible en $\mathcal{A} y$

$$
\int_{G} f(x) \mathrm{d} \mu(x)=\int_{\widehat{G}} f(x) \mathrm{d} \widehat{\mu}(x),
$$

donde $\widehat{\mu}: \widehat{G} \rightarrow[0, \infty]$ es la medida de Haar izquierda de $\widehat{G}$.

[Notemos que por el teorema de representación de RMK (teorema 1), la medida de Haar $\widehat{\mu}$ está totalmente determinada por las integrales de las funciones $f \in \mathcal{C}_{0}(\widehat{G})$. Lo que nos dice este teorema es que $\mu$ determina completamente a $\widehat{\mu}$.]

Demostración. El lema 12 nos garantiza que existe un grupo topológico localmente compacto $\widehat{G}$ del cual $G$ es un subgrupo denso. La siguiente afirmación nos permite conectar los conjuntos totalmente acotados de $G$ con los conjuntos compactos de $\widehat{G}$.

Afirmación: Si $E \subseteq G$ está contenido en un compacto de $\widehat{G}$, entonces $E$ es totalmente acotado en $G$.

En efecto, pensemos que $E \subseteq \widehat{C} \subseteq \widehat{G}$ con $\widehat{C}$ compacto, y sea $V \subseteq G$ una vecindad abierta de $e$. Denotemos por $\widehat{F} \subseteq \widehat{G}$ a la cerradura de $E$ en $\widehat{G}$. Entonces $\widehat{F}$ es compacto, pues es cerrado dentro del compacto $\widehat{C}$. Sea $\widehat{V} \subseteq \widehat{G}$ una vecindad abierta de $e$ tal que $\widehat{V} \cap G=V$. Si $y \in \widehat{F}$, como $y \widehat{V}^{-1}$ es una vecindad abierta de $y$, existe $x \in E \cap y \widehat{V}^{-1}$, y entonces $y \in x \widehat{V}$. Por lo tanto, la familia de conjuntos $x \widehat{V}$ con $x \in E$ forman una cubierta abierta del compacto $\widehat{F}$, y deben existir $x_{1}, x_{2}, \ldots, x_{n} \in E$ tales que 
$\widehat{F} \subseteq \bigcup_{i=1}^{n} x_{i} \widehat{V}$. Luego

$$
E \subseteq \widehat{F} \cap G \subseteq \bigcup_{i=1}^{n} x_{i} \widehat{V} \cap G=\bigcup_{i=1}^{n} x_{i} V,
$$

es decir, $E$ es totalmente acotado, demostrando la afirmación.

Para demostrar el resto del teorema, requeriremos del siguiente hecho sobre las funciones en $\mathcal{C}_{0}(\widehat{G})$. Toda $f \in \mathcal{C}_{0}(\widehat{G})$ es uniformemente continua por la izquierda (derecha), es decir, para todo $\varepsilon>0$ existe una vecindad $V$ de $e$ tal que para cada $x, y \in G$, si $x \in y V(V y)$, entonces $|f(x)-f(y)|<\varepsilon$. [10, 15.4, p. 185].

Tomemos $f \in \mathcal{C}_{0}(\widehat{G})$ y veamos que $\left.f\right|_{G}: G \rightarrow[0, \infty)$ es medible en $\mathcal{A}$, es decir, que para $A \in \mathcal{B}([0, \infty))$, se tiene $\left(\left.f\right|_{G}\right)^{-1}(A) \in \mathcal{A}$. Dado que $\mathcal{B}([0, \infty))$ es la $\sigma$-álgebra generada por los intervalos de la forma $[\alpha, \infty)$ con $\alpha \geq 0$, basta demostrar que $E \in \mathcal{A}$, donde $E=\left(\left.f\right|_{G}\right)^{-1}([\alpha, \infty))=\{x \in G \mid f(x)>\alpha\}$. En efecto, para cada $n \in \mathbb{N}$ definamos $E_{n}=\{x \in G \mid f(x) \geq \alpha+1 / n\}$. Como $f$ es uniformemente continua por la izquierda, existe una vecindad $W \subseteq G$ de $e$ que, sin pérdida de generalidad, está en $\mathcal{V}_{\mu} \subseteq \mathcal{A}$, tal que si $x \in y W$, entonces $|f(x)-f(y)|<1 / n$. Luego $E_{n} W \subseteq E$, pues si $y \in E_{n}$ y $x \in y W$, tenemos

$$
f(x)=f(y)+f(x)-f(y) \geq \alpha+\frac{1}{n}+f(x)-f(y) \geq \alpha+\frac{1}{n}-|f(x)-f(y)|>\alpha .
$$

Ahora, recordemos las funciones en $\mathcal{C}_{0}(\widehat{G})$ valen cero fuera de un conjunto compacto de $\widehat{G}$. Como $f \in \mathcal{C}_{0}(\widehat{G})$ y $f(x) \geq \alpha+1 / n>0$ para todo $x \in E_{n}$, tenemos que $E_{n}$ debe estar contenido en un conjunto compacto de $\widehat{G}$ y entonces la afirmación garantiza que $E_{n}$ es totalmente acotado en $G$. Sean $x_{1}, x_{2}, \ldots, x_{m} \in G$ tales que

$$
E_{n} \subseteq E_{n}^{\prime} \subseteq E_{n} W \subseteq E,
$$

donde $E_{n}^{\prime}=\bigcup_{i=1}^{m} x_{i} W \in \mathcal{A}$. Tenemos que

$$
E=\bigcup_{n \in \mathbb{N}} E_{n} \subseteq \bigcup_{n \in \mathbb{N}} E_{n}^{\prime} \subseteq E
$$

y entonces $E=\bigcup_{n \in \mathbb{N}} E_{n}^{\prime} \in \mathcal{A}$. Así, $\left.f\right|_{G}$ es medible en $\mathcal{A}$.

De nuevo, como $f \in \mathcal{C}_{0}(\widehat{G})$, el conjunto $S=\{x \in G \mid f(x) \neq 0\}$ debe de estar contenido en un conjunto compacto de $\widehat{G}$, y entonces, por la afirmación, $S$ es totalmente acotado en $G$. Por el lema $12, \mu(S)<\infty$, y entonces

$$
0 \leq \int_{G} f(x) \mathrm{d} \mu(x)=\int_{S} f(x) \mathrm{d} \mu(x) \leq \int_{S} M \mathrm{~d} \mu(x)=\mu(S) M<\infty,
$$

con $M=\operatorname{máx}\{f(x) \mid x \in S\}$. En consecuencia, podemos definir $I: \mathcal{C}_{0}(\widehat{G}) \rightarrow[0, \infty)$ por $I(f)=\int_{G} f(x) \mathrm{d} \mu(x)$. Es sencillo verificar que $I$ es una integral (i.e. es aditiva y homogénea). Por el teorema de representación de RMK (teorema 1), existe una única medida de Radon $\widehat{\mu}: \mathcal{B}(\widehat{G}) \rightarrow[0, \infty]$ tal que

$$
\int_{G} f(x) \mathrm{d} \mu(x)=\int_{\widehat{G}} f(x) \mathrm{d} \widehat{\mu}(x)
$$

para cada $f \in \mathcal{C}_{0}(\widehat{G})$.

Resta verificar que $\widehat{\mu}$ es una medida de Haar izquierda en $\widehat{G}$. Recordemos que por el teorema de representación de RMK (teorema 1), los valores de $\widehat{\mu}$ están completamente determinados por las integrales de las funciones en $\mathcal{C}_{0}(\widehat{G})$, y basta que veamos que

$$
\int_{\widehat{G}} f(\widehat{y} \widehat{x}) \mathrm{d} \widehat{\mu}(\widehat{x})=\int_{\widehat{G}} f(\widehat{x}) \mathrm{d} \widehat{\mu}(\widehat{x})
$$

para todo $\widehat{y} \in \widehat{G}$ y $f \in \mathcal{C}_{0}(\widehat{G})$.

Fijemos $f \in \mathcal{C}_{0}(\widehat{G})$. Si $\widehat{y}=y \in G$, definiendo $L_{y}: G \rightarrow G$ por $L_{y}(x)=y x$ y considerando la medida imagen $\mu L_{y}^{-1}: \mathcal{A} \rightarrow[0, \infty]$ (c.f. [4, 2.6.8, p. 76]), se obtiene 
$\mu L_{y}^{-1}(A)=\mu\left(y^{-1} A\right)=\mu(A)$ para cada $A \in \mathcal{A}$ (por la invarianza izquierda de $\mu$ ), y entonces

$$
\begin{aligned}
\int_{\widehat{G}} f(y \widehat{x}) \mathrm{d} \widehat{\mu}(\widehat{x}) & =\int_{G} f(y x) \mathrm{d} \mu(x)=\int_{G}\left(f \circ L_{y}\right)(x) \mathrm{d} \mu(x)=\int_{G} f(x) \mathrm{d}\left(\mu L_{y}^{-1}\right)(x) \\
& =\int_{G} f(x) \mathrm{d} \mu(x)=\int_{\widehat{G}} f(\widehat{x}) \mathrm{d} \widehat{\mu}(\widehat{x}) .
\end{aligned}
$$

Para $\widehat{y} \notin G$, debemos hacer un poco más de trabajo. Tomemos $\varepsilon>0$ y veamos que

$$
\left|\int_{\widehat{G}} f(\widehat{y} \widehat{x}) \mathrm{d} \widehat{\mu}(\widehat{x})-\int_{\widehat{G}} f(\widehat{x}) \mathrm{d} \widehat{\mu}(\widehat{x})\right| \leq \varepsilon .
$$

Como $\widehat{G}$ es localmente compacto, existe una vecindad compacta $\widehat{C} \subseteq \widehat{G}$ de $e$, y como $f \in \mathcal{C}_{0}(\widehat{G})$, existe un compacto $\widehat{S} \subseteq \widehat{G}$ tal que $f(\widehat{x})=0$ si $\widehat{x} \notin \widehat{S}$. El conjunto

$$
\widehat{N}=\widehat{y}^{-1} \widehat{C} \widehat{S}
$$

es compacto (el producto de compactos es compacto [10, 4.4, p. 17]). Como $\widehat{\mu}$ es una medida de Radon, es localmente finita [ver teorema 1](I)], y entonces $\widehat{\mu}(\widehat{N})<\infty$. Sea $\delta>0$ tal que $\delta \widehat{\mu}(\widehat{N}) \leq \varepsilon$. Como $f$ es uniformemente continua por la derecha, existe una vecindad $\widehat{V} \subseteq \widehat{G}$ de $e$ tal que

$$
|f(\widehat{v})-f(\widehat{w})|<\delta \quad \text { si } \quad \widehat{w} \in \widehat{V} \widehat{v} .
$$

Sin pérdida de generalidad, podemos suponer que $\widehat{V} \subseteq \widehat{C}^{-1}$. Como $G$ es denso, existe $y \in G \cap \widehat{V} \widehat{y}$ y, entonces, para cada $\widehat{x} \in G$ tenemos $y \widehat{x} \in \widehat{V} \widehat{y} \widehat{x}$, de donde obtenemos que $|f(\widehat{y} \widehat{x})-f(y \widehat{x})|<\delta$.

Además, notemos que si $\widehat{x} \notin \widehat{N}$, entonces $\widehat{y} \widehat{x} \notin \widehat{C} \widehat{S} \supseteq \widehat{S}$, por lo que $f(\widehat{y} \widehat{x})=0$, y también

$$
y \widehat{x} \notin y \widehat{y}^{-1} \widehat{C} \widehat{S} \supseteq y \widehat{y}^{-1} \widehat{V}^{-1} \widehat{S} \supseteq \widehat{S}, \quad \text { (pues } e \in y \widehat{y}^{-1} \widehat{V}^{-1}, \text { ya que } y \in \widehat{V} \widehat{y} \text { ) }
$$

por lo que $f(y \widehat{x})=0$. Por lo tanto, $|f(\widehat{y} \widehat{x})-f(y \widehat{x})|=0$ para todo $\widehat{x} \notin \widehat{N}$ y entonces

$$
\begin{aligned}
\left|\int_{\widehat{G}} f(\widehat{y} \widehat{x}) \mathrm{d} \widehat{\mu}(\widehat{x})-\int_{\widehat{G}} f(\widehat{x}) \mathrm{d} \mu(\widehat{x})\right| & =\left|\int_{\widehat{G}} f(\widehat{y} \widehat{x}) \mathrm{d} \widehat{\mu}(\widehat{x})-\int_{\widehat{G}} f(y \widehat{x}) \mathrm{d} \widehat{\mu}(\widehat{x})\right| \\
& \leq \int_{\widehat{G}}|f(\widehat{y} \widehat{x})-f(y \widehat{x})| \mathrm{d} \widehat{\mu}(\widehat{x}) \\
& =\int_{\widehat{N}}|f(\widehat{y} \widehat{x})-f(y \widehat{x})| \mathrm{d} \widehat{\mu}(\widehat{x}) \\
& \leq \int_{\widehat{N}} \delta \mathrm{d} \widehat{\mu}(\widehat{x})=\delta \widehat{\mu}(\widehat{N}) \leq \varepsilon .
\end{aligned}
$$

\section{Agradecimientos}

Agradezco a la Dra. Natalia Jonard Pérez por sus detallados comentarios, a la Dra. Judith Campos Cordero por haberme invitado a realizar este artículo y al árbitro anónimo por sus valiosas correcciones.

\section{REFERENCIAS}

[1] Arkhangelskii, A. V. \& Tkachenko, M., Topological Groups and Related Structures. Atlantis Press, Francia, 2008, ISBN 9789491216350.

[2] Bredon, G. E., A new treatment of the Haar integral. The Michigan Math. Jour., 10, no. 4 365-373, 1963, DOI:10.1307/mmj/1028998972, https://projecteuclid.org/euclid.mmj/1028998972

[3] Cartan, H., Sur la mesure de Haar. C. R. Acad. Sci. Paris, 211, 759-762, 1940.

[4] Cohn, D., Measure Theory. Birkhäuser, Estados Unidos, $2^{\text {a }}$ ed., 2013, ISBN 9781461469551.

[5] Diestel, J. \& Spalsbury, A., The Joys of Haar Measure. American Mathematical Society, Estados Unidos, 2014, ISBN 9781470414115.

[6] Dudley, R. M., Real Analysis and Probability. Cambridge University Press, Cambridge New York, 2002, ISBN 0521007542.

[7] Folland, G. B., Real Analysis: Modern Techniques and Their Applications. PAM, Wiley, $2^{\text {a }}$ ed., 1999, ISBN 9780471317166. 
[8] Haar, A., Der Massbegriff in der Theorie der Kontinuierlichen Gruppen. The Ann. of Math., 34, no. 1, 147, 1933, DOI:10.2307/1968346, https://www.jstor.org/stable/1968346.

[9] Halmos, P., Measure Theory. Springer Science, Estados Unidos, 1950, ISBN 9780387900889.

[10] Hewitt, E. \& Ross, K. A., Abstract Harmonic Analysis, vol. 1. Springer, Estados Unidos, 1979, ISBN 9780387941905.

[11] Pettis, B. J., On continuity and openness of homomorphisms in topological groups. The Ann. of Math, 52, no. 2, 293, 1950, DOI:10.2307/1969471, https://www.jstor.org/stable/1969471

[12] Pilatowsky Cameo, S., El teorema de Haar y su inverso. Tesis de Licenciatura, Universidad Nacional Autónoma de México, Ciudad Universitaria, 2021. Disponible en TESIUNAM: https://tesiunam.dgb.unam.mx/F/?func=service\&doc_number=000811324\&service_type=MEDIA

[13] Rudin, W., Real and Complex Analysis. McGraw-Hill, Estados Unidos, 1987, ISBN 9780070542341.

[14] Weil, A., Sur les Espaces à Structure Uniforme et sur la Topologie Générale. Hermann, Francia, 1937.

[15] Weil, A., L'Intégration dans les Groupes Topologiques et ses Applications. Hermann, Francia, 1940, ISBN 9782705611453.

Dirección del autor:

Facultad de Ciencias,

Universidad Nacional Autónoma de México,

Ciudad Universitaria, 04510 CDMX.

ORCID: https://orcid.org/0000-0001-9843-6491

e-mail: saulpilatowsky@ciencias.unam.mx 